\title{
Impact of prognostic nutritional index on the recurrence of hepatocellular carcinoma after a curative resection
}

\author{
Ho Jeong, Kil Hwan Kim, Sungho Jo, Sanghyun Song
}

Department of Surgery, Dankook University Hospital, Dankook University College of Medicine, Cheonan, Korea

\begin{abstract}
Backgrounds/Aims: The purpose of this retrospective study was to determine the association between prognostic nutritional index (PNI) and recurrence of hepatocellular carcinoma after a curative resection.

Methods: Between 2007 to 2019, 130 patients who underwent curative hepatectomy for hepatocellular carcinoma were enrolled. PNI was calculated. Its cutoff value was identified through receiver operating characteristic curve analysis. According to PNI, patients were divided into two groups. Univariate and multivariate analyses were performed to identify independent risk factors for recurrence.

Results: The cutoff value of PNI was 52. In univariate analysis, alcoholic liver cirrhosis $(p=0.041)$, protein induced by vitamin $\mathrm{K}$ antagonist-II $\geq 200(p=0.012)$, indocyanine green retention test (ICG R15) $>10 \%(p=0.001)$, estimated blood loss $\geq 800 \mathrm{~mL}(p=0.037)$, tumor size $(p=0.001)$, microvascular invasion $(p=0.023)$, T-stage $(p=0.001)$, and PNI $<52(p=0.001)$ were significant factors affecting the recurrence. In multivariate analysis, alcoholic liver cirrhosis $(p=0.046)$, ICG R15 $>10 \%(p=0.025)$, T-stage $(p=0.003)$, and PNI $<52(p=0.046)$ were independent prognostic factors for disease-free survival.

Conclusions: PNI, a nutritional and immunologic factor, is an independent prognostic factor that can predict the recurrence of hepatocellular carcinoma in patients undergoing a curative resection.
\end{abstract}

Key Words: Carcinoma, hepatocellular; Nutrition assessment; Recurrence

\section{INTRODUCTION}

Hepatocellular carcinoma (HCC) is the 6th most common malignancy worldwide and the 2nd leading cause of cancer death [1]. According to the annual report of cancer statistics in Korea in 2016, HCC was the 6th most common cancer. However, its mortality rate was the second highest. There are many treatment modalities such as transarterial chemoembolization (TACE), radiofrequency ablation (RFA), percutaneous ethanol injection, and radiation. However, curative resection and liver transplantation are still considered first-line treatment options [2]. Its long-term prognosis remains unsatisfactory. Its 5-year

Received: January 31, 2021, Revised: April 1, 2021,

Accepted: April 7, 2021

Corresponding author: Sanghyun Song

Department of Surgery, Dankook University Hospital, Dankook University College of Medicine, 119 Dandae-ro, Dongnam-gu, Cheonan 31116, Korea Tel: +82-41-550-3087, Fax: +82-41-550-6034, E-mail: youngmoon77@gmail.com ORCID: https://orcid.org/0000-0002-9208-9537

Copyright (C) The Korean Association of Hepato-Biliary-Pancreatic Surgery

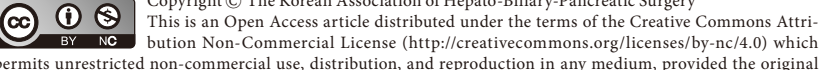

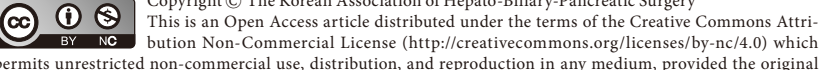
permits unrestricted non
work is properly cited. recurrence rate is approximately $50 \%-70 \%$ even after a curative resection $[3,4]$. The high recurrence incidence remains a major cause of death after a curative resection. Its well-known prognostic factors up to date include microvascular invasion, poor differentiation, multiple tumors, and tumor size [5-7]. Most patients with HCC have chronic liver diseases such as hepatitis B virus (HBV), hepatitis $\mathrm{C}$ virus (HCV), and alcoholic liver cirrhosis. It is difficult to predict the prognosis of patients with only the tumor burden as prognosis is affected by impaired liver function related to underlying liver disease. Many studies have confirmed that the pathogenesis of HCC is associated with inflammation of the underlying chronic liver disease. Inflammation is an essential factor in tumor progression [8]. In addition, cancer cachexia has been observed in many patients with HCC. Furthermore, the nutritional status of patients is closely related to the function of the immune system and the inflammatory response. Initially, prognostic nutritional index (PNI) has been proposed to assess immunological and nutritional aspects of patients who undergo a gastrointestinal surgery [9]. Preoperative PNI can be calculated as follows: $10 \times$ serum albumin $(\mathrm{g} / \mathrm{dL})+0.005 \times$ total lymphocyte count $($ per $\mathrm{mm}^{3}$ ). Many studies have indicated that PNI is a prognostic 
factor for gastrointestinal malignancies [10]. However, there are limited data on screening HCC patients for malnutrition. Little data has been released on the prognostic value of PNI in these patients. Therefore, PNI was used in this retrospective cohort study of patients with HCC. It was found that PNI was a significant predictor of recurrence after a curative resection.

\section{PATIENTS AND METHODS}

\section{Patients}

Clinical data of patients who underwent hepatectomy for HCC between January 2007 and December 2019 at Dankook University Hospital were retrospectively reviewed. Only patients with complete follow-up information were included. Patients with evidence of infection or systemic inflammatory diseases were excluded. Patients who underwent locoregional therapy such as TACE and RFA before surgery and those who had HCC recurrence were also excluded. In addition, patients with combined hepatocellular cholangiocarcinoma were excluded from postoperative biopsy results. Five in-hospital deaths were also excluded. Curative hepatectomy was defined as complete removal of HCC without grossly or microscopically identifiable tumor residue on the resection margin. We collected data from clinical records, including basic patient demographics, concomitant diseases, complete blood count, biochemistry and liver function parameters, alpha-fetoprotein (AFP), prothrombin induced by vitamin $\mathrm{K}$ absence or antagonist-II (PIVKA-II), HBV and HCV markers, T-stage, clinicopathologic parameters, and other tumor-related parameters for each patient. Detailed medical history, physical examination, and preoperative routine laboratory tests were performed at least 7 days prior to the surgery. The diagnosis of HCC was made based on preoperative radiological examination criteria and confirmed by postoperative pathological findings. Of 130 patients, 52 underwent major resections (over three segments) and 78 patients underwent minor resections. There were 84 anatomical resections and 46 non-anatomical resections.

\section{Follow-up}

Regular follow-up was conducted in the outpatient clinic. Monthly serum AFP and PIVKA-II tests were performed during the first three months after surgery. Chest radiography and contrast-enhanced computed tomography were performed at least every three months for the first two years. Patients were followed up with serological and imaging tests every six months during the first three to five years. Five years later, follow-up was performed annually. Tumor recurrence was diagnosed using imaging and laboratory tests. Patients with confirmed HCC recurrence underwent individualized treatment, including repeated liver resection, TACE, RFA, and liver transplantation. The median follow-up was 34.5 months (range, 2-157 months). The last follow-up date was in July 2020.

\section{Ethical considerations}

The study protocol was reviewed and approved by the Institutional Review Board (IRB) of Dankook University Hospital (IRB No: DKUH 2021-01-017). The requirement for informed consent was waived because of the retrospective nature of this study.

\section{Statistical analysis}

To assess the sensitivity and specificity of PNI for predicting recurrence, we analyzed receiver operating characteristic (ROC) curve and identified the optimum cutoff value for PNI. We divided patients into two groups according to the cutoff value of the PNI. Continuous variables were represented as the median interquartile range and compared with a Mann-Whitney U-test. Categorical variables were expressed as counts (percentages) and compared with chi-square test or Fisher's exact test when appropriate. Factors associated with recurrence-free survival were analyzed using univariate and multivariate logistic regression analyses. Disease-free survival (DFS) curve was calculated using the Kaplan-Meier method and compared using a log-rank test. All statistical analyses were performed using IBM SPSS version 26 (IBM Corp., Armonk, NY, USA).

\section{RESULTS}

The area under the ROC curve was 0.572 . The optimal cutoff value of PNI was 51.85 (Fig. 1). Therefore, the optimum PNI cutoff value was set at 52 . We defined 53 patients with PNI $\geq$ 52 as the high-PNI group and 77 patients with PNI $<52$ as the low-PNI group. Associations between clinicopathological parameters and PNI are shown in Table 1. Albumin levels were higher in the high-PNI group with improved coagulation function. Results of indocyanine green retention test (ICG R15) for

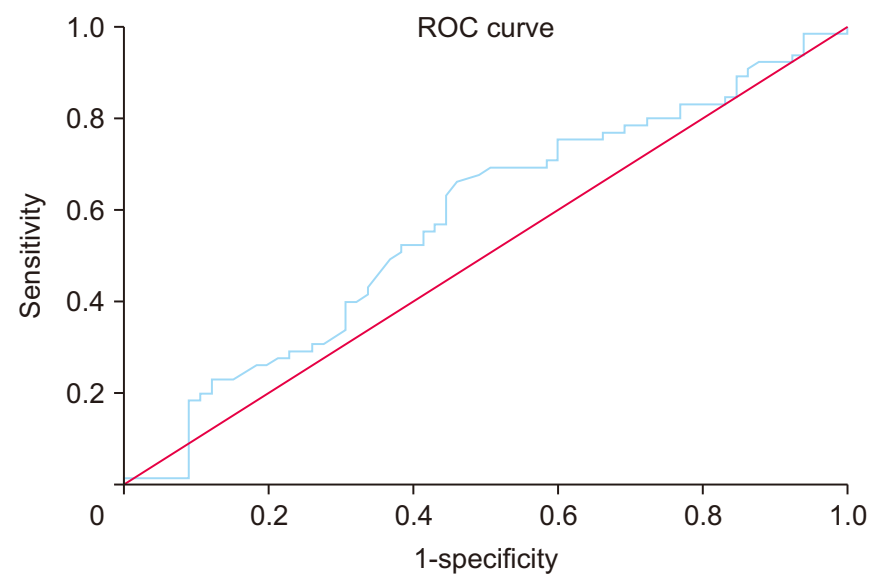

Fig. 1. Receiver operating characteristic (ROC) curve. The area under the ROC curve is 0.572 (95\% confidence interval: $0.47-0.67, p<0.05$ ) and the optimal cutoff value is 51.85 . 
Table 1. Characteristics of patients included in this study

\begin{tabular}{|c|c|c|c|}
\hline Variable & PNI-high group $(n=53)$ & PNI-low group $(n=77)$ & $p$-value \\
\hline Age (yr) & $54(33-80)$ & $61(39-83)$ & 0.187 \\
\hline Male & $44(83.0)$ & $67(87.0)$ & 0.616 \\
\hline Hepatitis & & & 0.097 \\
\hline HBV & $41(77.4)$ & $44(57.1)$ & \\
\hline $\mathrm{HCV}$ & $3(5.7)$ & $7(9.1)$ & \\
\hline Alcohol & $3(5.7)$ & $13(16.9)$ & \\
\hline Child-Pugh & & & 0.121 \\
\hline A & $51(96.2)$ & $67(87.0)$ & \\
\hline B & $2(3.8)$ & $10(13.0)$ & \\
\hline \multicolumn{4}{|l|}{ Comorbidity } \\
\hline Diabetes & $42(79.2)$ & $50(64.9)$ & 0.116 \\
\hline Hypertension & $34(64.2)$ & $48(62.3)$ & 0.855 \\
\hline Albumin (g/dL) & $4.4(3.7-5.2)$ & $3.9(2.3-4.8)$ & $<0.001$ \\
\hline Lymphocyte $\left(/ \mathrm{mm}^{3}\right)$ & $2,380(1,650-6,760)$ & $1,520(490-2,620)$ & $<0.001$ \\
\hline Platelet $\left(\times 10^{3} / \mathrm{mm}^{3}\right)$ & $170(79-345)$ & $156(56-464)$ & 0.913 \\
\hline PTINR & $0.97(0.86-1.85)$ & $1.03(0.83-1.61)$ & 0.036 \\
\hline $\mathrm{CRP}(\mathrm{mg} / \mathrm{dL})$ & $0.15(0.01-3.02)$ & $0.26(0.01-3.89)$ & 0.150 \\
\hline $\mathrm{ALT}(\mathrm{U} / \mathrm{L})$ & $29(10-164)$ & $31(4-137)$ & 0.747 \\
\hline Total bilirubin (mg/dL) & $0.5(0.19-2.58)$ & $0.6(0.17-1.93)$ & 0.455 \\
\hline $\operatorname{AFP}(\mathrm{ng} / \mathrm{mL})$ & $20.3(0.6-15,000)$ & $7.2(0.5-11,340)$ & 0.339 \\
\hline PIVKA-II (mAU/mL) & $58(10-33,726)$ & $77(9-48,555)$ & 0.157 \\
\hline ICG R15 (\%) & $8.3(1-22.7)$ & $11.5(1-32)$ & 0.079 \\
\hline Estimated blood loss $(\mathrm{mL})$ & $400(0-2,400)$ & $600(0-5,000)$ & 0.017 \\
\hline Blood loss $>800 \mathrm{~mL}$ & $11(20.8)$ & $28(36.4)$ & 0.079 \\
\hline Intraoperative transfusion & $6(11.3)$ & $29(37.7)$ & 0.001 \\
\hline Operation time (min) & $260(95-580)$ & $230(65-985)$ & 0.419 \\
\hline \multicolumn{4}{|l|}{ Operation method } \\
\hline Laparoscopic & $23(43.4)$ & $23(29.9)$ & 0.137 \\
\hline Nonanatomic resection & $17(32.1)$ & $29(37.7)$ & 0.692 \\
\hline Major resection ${ }^{a)}$ & $24(45.3)$ & $28(36.4)$ & 0.364 \\
\hline Size $(\mathrm{cm})$ & $2.7(0.8-10)$ & $3.5(1-24)$ & 0.012 \\
\hline Margin (mm) & $11(1-70)$ & $10(1-55)$ & 0.367 \\
\hline Multiple tumor & $9(17.0)$ & $8(10.4)$ & 0.299 \\
\hline Edmondson grade & & & 0.398 \\
\hline Grade 1 & $4(7.5)$ & $10(13.0)$ & \\
\hline Grade 2-4 & $49(92.5)$ & $67(87.0)$ & \\
\hline Microvascular invasion & $13(24.5)$ & $16(20.8)$ & 0.671 \\
\hline T-stage & & & 0.055 \\
\hline 1 & $31(58.5)$ & $41(53.2)$ & \\
\hline 2 & $18(34.0)$ & $17(22.1)$ & \\
\hline 3 & 0 & $4(5.2)$ & \\
\hline 4 & $4(7.5)$ & $15(19.5)$ & \\
\hline Recurrence & $17(32.1)$ & $48(62.3)$ & 0.001 \\
\hline
\end{tabular}

Values are presented as median (interquartile range) or number (\%).

PNI, prognostic nutritional index; HBV, hepatitis B virus; $\mathrm{HCV}$, hepatitis C virus; PT INR, prothrombin time international normalized ratio; CRP, C-reactive protein; ALT, alanine aminotransferase; AFP, alpha fetoprotein; PIVKA II, prothrombin induced by vitamin K antagonist-II; ICG R15, indocyanine green retention test.

${ }^{\text {a) }}$ Resection $>3$ segments. 
predicting residual liver function before surgery were found to be better in the high-PNI group than those in the low-PNI group, although the difference between the two groups was not significant. In the low-PNI group, there was more bleeding during surgery and more frequent blood transfusions. The tumor size was smaller in the high-PNI group. HCC recurred in $65(50.0 \%)$ patients, including 17 patients $(32.1 \%)$ in the highPNI group and 48 patients (62.3\%) in the low-PNI group.

A univariate analysis was performed to investigate factors affecting HCC recurrence. In the univariate analysis, alcoholic liver cirrhosis, PIVKA-II $\geq 200$, ICG R15 > 10\%, estimated

Table 2. Univariate analysis of disease-free survival

\begin{tabular}{|c|c|c|c|}
\hline Variable & $\mathrm{HR}$ & $95 \% \mathrm{Cl}$ & $p$-value \\
\hline Age $(\geq 70 /<70 \mathrm{yr})$ & 0.82 & $0.34-1.95$ & 0.661 \\
\hline Sex (male/female) & 1.00 & $0.19-1.45$ & 0.601 \\
\hline $\operatorname{HBV}(+/-)$ & 0.62 & $0.29-1.28$ & 0.198 \\
\hline $\mathrm{HCV}(+/-)$ & 1.55 & $0.41-5.77$ & 0.513 \\
\hline Alcohol (+/-) & 3.45 & $1.05-11.34$ & 0.041 \\
\hline Child-Pugh (B/A) & 1.44 & $0.43-4.82$ & 0.546 \\
\hline Diabetes (+/-) & 1.82 & $0.84-3.93$ & 0.125 \\
\hline Hypertension (+/-) & 1.70 & $0.82-3.49$ & 0.147 \\
\hline Albumin & 0.72 & $0.34-1.51$ & 0.392 \\
\hline Lymphocyte & 1.00 & $0.99-1.00$ & 0.350 \\
\hline Platelet $\left(/ \mathrm{mm}^{3}\right)\left(\leq 1.0 \times 10^{5} />1.0 \times 10^{5}\right)$ & 1.16 & $0.39-3.41$ & 0.784 \\
\hline PT INR $(>1.2 / \leq 1.2)$ & 3.20 & $0.62-16.50$ & 0.164 \\
\hline $\mathrm{CRP}(\mathrm{mg} / \mathrm{dL})$ & 1.16 & $0.71-1.89$ & 0.535 \\
\hline $\operatorname{ALT}(\mathrm{U} / \mathrm{L})$ & 1.01 & $0.99-1.02$ & 0.088 \\
\hline Total bilirubin (mg/dL) & 1.46 & $0.60-3.59$ & 0.400 \\
\hline $\operatorname{AFP}(\geq 40 /<40)$ & 1.61 & $0.77-3.34$ & 0.198 \\
\hline PIVKA-II $(\geq 200 /<200)$ & 2.57 & $1.23-5.37$ & 0.012 \\
\hline ICG R15 (> 10\%/ $10 \%)$ & 3.86 & $1.71-8.76$ & 0.001 \\
\hline $\begin{array}{l}\text { Estimated blood loss } \\
\qquad(\geq 800 \mathrm{~mL} /<800 \mathrm{~mL})\end{array}$ & 2.27 & $1.05-4.93$ & 0.037 \\
\hline Intraoperative transfusion (+/-) & 1.73 & $0.79-3.82$ & 0.169 \\
\hline Operation time ( $\geq 300 \mathrm{~min} /<300 \mathrm{~min}$ ) & 1.65 & $0.78-3.49$ & 0.188 \\
\hline Laparoscopic resection (+/-) & 0.58 & $0.28-1.20$ & 0.144 \\
\hline Non-anatomical resection (+/-) & 1.10 & $0.59-2.08$ & 0.748 \\
\hline Major resection $^{\text {a) }}(+/-)$ & 1.67 & $0.82-3.40$ & 0.153 \\
\hline Size $(\mathrm{cm})$ & 1.23 & $1.08-1.40$ & 0.001 \\
\hline Margin $(\leq 10 \mathrm{~mm} />10 \mathrm{~mm})$ & 0.93 & $0.46-1.90$ & 0.857 \\
\hline Multiple tumor (+/-) & 2.00 & $0.69-5.78$ & 0.199 \\
\hline Edmondson grade (1/2-4) & 1.38 & $0.45-4.22$ & 0.573 \\
\hline Microvascular invasion (+/-) & 2.76 & $1.14-6.66$ & 0.023 \\
\hline T-stage & 2.07 & $1.38-3.10$ & 0.001 \\
\hline PNI $(\geq 52 /<52)$ & 3.50 & $1.67-7.33$ & 0.001 \\
\hline
\end{tabular}

$\mathrm{HR}$, hazard ratio; $\mathrm{Cl}$, confidence interval; $\mathrm{HBV}$, hepatitis $\mathrm{B}$ virus; $\mathrm{HCV}$, hepatitis $C$ virus; PT INR, prothrombin time international normalized ratio; CRP, C-reactive protein; ALT, alanine aminotransferase; AFP, alpha fetoprotein; PIVKA-II, prothrombin induced by vitamin K antagonist-II; ICG R15, indocyanine green retention test; PNI, prognostic nutritional index.

${ }^{\text {a) }}$ Resection $>3$ segments. blood loss $\geq 800 \mathrm{~mL}$, tumor size, microvascular invasion, T-stage, and PNI $<52$ were closely related to HCC recurrence after a curative resection (Table 2). Multivariate analysis showed that alcoholic liver cirrhosis (hazard ratio [HR]: 4.33, 95\% confidence interval [CI]: 1.02-18.28, $p=0.046)$, ICG R15 $>10 \%$ (HR: $1.10,95 \%$ CI: $1.01-1.20, p=0.025)$, T-stage (HR: 2.20, 95\% CI: $1.31-3.70, p=0.003$ ), and PNI < 52 (HR: 2.60, 95\% CI: $1.01-6.68, p=0.046)$ were independently associated with HCC recurrence after a curative resection (Table 3). Cumulative DFS was estimated using the Kaplan-Meier method (Fig. 2). One-year, 3-year, and 5-year cumulative DFS rates were $92.1 \%, 69.7 \%$, and $69.7 \%$ in the high-PNI group and $89.3 \%$, $63.3 \%$, and $40.2 \%$ in the low-PNI group, respectively $(p=0.011)$.

\section{DISCUSSION}

Surgical resection for HCC is considered a safe operation due to its low mortality and morbidity. However, the prognosis of

Table 3. Multivariate analysis of disease-free survival

\begin{tabular}{lllc}
\hline \multicolumn{1}{c}{ Variable } & HR & \multicolumn{1}{c}{$95 \% \mathrm{Cl}$} & $p$-value \\
\hline Alcohol $(+/-)$ & 4.33 & $1.02-18.28$ & 0.046 \\
PIVKA-II $(\geq 200 /<200)$ & 1.03 & $0.34-3.13$ & 0.948 \\
ICG R15 $(>10 \% / \leq 10 \%)$ & 1.10 & $1.01-1.20$ & 0.025 \\
Estimated blood loss & 1.45 & $0.52-4.06$ & 0.476 \\
$\quad(\geq 800 \mathrm{~mL} /<800 \mathrm{~mL})$ & & & \\
Size $(\mathrm{cm})$ & 1.00 & $0.84-1.19$ & 0.940 \\
Microvascular invasion $(+/-)$ & 2.24 & $0.70-7.20$ & 0.174 \\
T-stage & 2.20 & $1.31-3.70$ & 0.003 \\
PNI $(\geq 52 /<52)$ & 2.60 & $1.01-6.68$ & 0.046 \\
\hline
\end{tabular}

$\mathrm{HR}$, hazard ratio; $\mathrm{Cl}$, confidence interval; PIVKA-II, prothrombin induced by vitamin $\mathrm{K}$ antagonist-II; ICG R15, indocyanine green retention test; PNI, prognostic nutritional index.

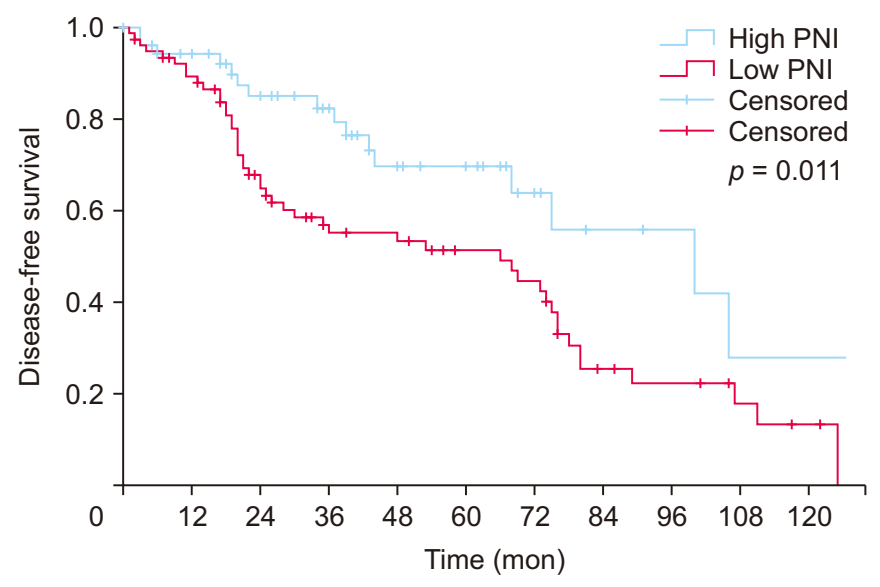

Fig. 2. Disease-free survival curves of patients in the high-PNI group compared to those in the low-PNI group. PNI, prognostic nutritional index. 
patients is poor because of the high recurrence rate after curative resection. Therefore, understanding and predicting recurrence are important for improving the prognosis of patients with HCC. There are three categories of factors involved in the recurrence after a curative resection of HCC [1]: 1) tumor factors such as tumor size, number, Edmondson-Steiner grade, and preoperative tumor markers [2]; 2) host factors such as the grade of hepatitis activity, presence of cirrhosis, and hepatitis viral load [3]; and 3) surgical factors such as surgical margin and extent of liver resection [11]. There have been many studies on tumor factors. Microvascular invasion is a predictor of tumor recurrence after curative resection. High AFP and multiple tumors also affect prognosis $[5,6]$. In addition, some studies have reported that HCV-related HCC has a poorer prognosis than HBV-related HCC [4]. It has been reported that satellite nodule, $\mathrm{R} 1$ margin resection, and non-anatomical resection can adversely affect recurrence [3]. In the present study, T-stage was found to be a significant risk factor for tumor recurrence after a curative resection.

Inflammatory responses play decisive roles at different stages of tumor development, including initiation, promotion, malignant conversion, invasion, and metastasis [8]. HCC was associated with $\mathrm{HBV}$ or $\mathrm{HCV}$ in most patients [8]. In that study, 95 of 130 patients (73\%) were HBV or HCV related [8]. In our study, among 16 patients with alcoholic liver cirrhosis, $85 \%$ of patients had chronic hepatitis. In HCC patients, chronic inflammation might affect the recurrence and/or prognosis of cancer. Some studies have shown that these factors are linked to prognosis. Lymphocytes are immune factors of PNI. These cells play an important role in the recurrence and progression of HCC. A clinical study on resected specimens of HCC patients has shown that high densities of CD3(+) and CD8(+) T cells in the interior and margins of the tumor are significantly associated with a low rate of recurrence and a prolonged DFS [12]. These findings suggest that the host's immune status such as lymphocyte infiltration into the tumor might play an important role in preventing tumor progression. The lymphocyte-to-monocyte ratio can predict survival outcomes in HBV-related HCC patients who undergo a curative hepatectomy [13]. The neutrophil-to-lymphocyte ratio and aspartate aminotransferase to platelet ratio can also predict recurrence after a curative resection $[14,15]$. In addition to the lymphocyte scoring system, the modified Glasgow prognostic score (mGPS) is a score that can confirm host's inflammatory response. It is a combination of C-reactive protein and albumin levels. Indeed, a large cohort study has reported that mGPS can predict cancer survival independent of tumor sites [16]. According to these studies, the host inflammatory response can affect both recurrence and survival.

Nutrition has been linked to the prognosis of malignant tumors. Malnutrition has also been reported as an important prognostic factor in patients with HCC [17]. Furthermore, malnutrition is a risk factor for postoperative complications after hepatectomy in patients with HCC [18]. Although there are several scoring systems to determine the patient's nutritional status before surgery, PNI is simple. PNI consists of albumin level and lymphocytes, which may reflect a balance between pro-tumor inflammatory status and nutritional status. In addition, albumin, a component of PNI, can reflect protein synthesis disorders following a chronic liver disease of HCC. In a meta-analysis, preoperative PNI is also a prognostic marker in patients with HCC $[19,20]$. According to the study by Ji et al. [21], PNI and body mass index can be used as important biomarkers to screen patients with early stage HCC for high risk of recurrence and death. One study has shown that PNI is a useful marker to predict survival of HCC patients treated with sorafenib [22]. Moreover, postoperative PNI, not preoperative PNI, could predict survival of patients with HCC after a surgical resection [23]. In the present study, PNI was confirmed to be an independent predictor of recurrence after a curative resection with alcoholic liver cirrhosis, ICG R15, and T-stage. In particular, alcoholic liver cirrhosis was identified as an independent prognostic factor. Most alcoholic liver cirrhosis patients are thought to be have poor nutrition.

ICG R15 is a common parameter for preoperative assessment of preserved hepatic function [24]. ICG R15 has been reported to be an early indicator of hepatic dysfunction. It has been used preoperatively to plan the extent of partial hepatectomy by predicting the risk of dysfunction after surgery. ICG R15 with a cutoff value of $10 \%$ as an upper limit of normal has been widely used in clinical practice in Korea. In the present study, ICG R15 > 10\% was identified as an independent prognostic factor for DFS. As albumin, lymphocyte count, and ICG R15 are routine tests performed in clinical practice, the assessment of their values can be used to predict recurrence after surgery.

Our study has some limitations. First, the sample size was small because this was a retrospective single-center study. Second, selection bias was inevitable. It might have influenced the analysis because we selected only patients who had undergone a curative resection. Third, PNI did not affect survival. We investigated the survival curve using the Kaplan-Meier method. The $p$-value was 0.067 , which was not statistically significant. Thus, large-scale studies should be conducted in the future.

In summary, our study examined risk factors affecting recurrence after curative resection for HCC. Patients with preoperative low-PNI, ICG R15 > 10\%, alcoholic liver cirrhosis, and advanced T-stage had more recurrence after a curative resection. Our findings could be used to identify patients at risk of recurrence after hepatectomy and help them receive necessary treatment to improve their survival. However, more extensive cohort studies need to be conducted in the future to validate the clinical usefulness of PNI and other factors as predictive markers for recurrence in HCC. 


\section{CONFLICT OF INTEREST}

No potential conflict of interest relevant to this article was reported.

\section{ORCID}

Ho Jeong, https://orcid.org/0000-0001-7035-1832

Kil Hwan Kim, https://orcid.org/0000-0002-7841-4030

Sungho Jo, https://orcid.org/0000-0002-5004-0903

Sanghyun Song, https://orcid.org/0000-0002-9208-9537

\section{AUTHOR CONTRIBUTIONS}

Conceptualization: SS. Data curation: HJ, SS. Methodology: KHK, SJ. Writing - original draft: HJ. Writing - review \& editing: SS.

\section{REFERENCES}

1. Ferlay J, Soerjomataram I, Dikshit R, Eser S, Mathers C, Rebelo $\mathrm{M}$, et al. Cancer incidence and mortality worldwide: sources, methods and major patterns in GLOBOCAN 2012. Int J Cancer 2015;136:E359-E386.

2. European Association For The Study Of The Liver; European Organisation For Research And Treatment Of Cancer. EASL-EORTC clinical practice guidelines: management of hepatocellular carcinoma. J Hepatol 2012;56:908-943.

3. Muscari F, Foppa B, Carrere N, Kamar N, Peron JM, Suc B. Resection of a transplantable single-nodule hepatocellular carcinoma in ChildPugh class A cirrhosis: factors affecting survival and recurrence. World J Surg 2011;35:1055-1062.

4. Naito S, Imamura H, Tukada A, Matsuyama Y, Yoshimoto J, Sugo H, et al. Postoperative recurrence pattern and prognosis of patients with hepatocellular carcinoma, with particular reference to the hepatitis viral infection status. Liver Int 2014;34:802-813.

5. Lim KC, Chow PK, Allen JC, Chia GS, Lim M, Cheow PC, et al. Microvascular invasion is a better predictor of tumor recurrence and overall survival following surgical resection for hepatocellular carcinoma compared to the Milan criteria. Ann Surg 2011;254:108-113.

6. Zhu WJ, Huang CY, Li C, Peng W, Wen TF, Yan LN, et al. Risk factors for early recurrence of HBV-related hepatocellular carcinoma meeting milan criteria after curative resection. Asian Pac J Cancer Prev 2013;14:7101-7106.

7. Park SK, Jung YK, Chung DH, Kim KK, Park YH, Lee JN, et al. Factors influencing hepatocellular carcinoma prognosis after hepatectomy: a single-center experience. Korean J Intern Med 2013;28:428-438.

8. Grivennikov SI, Greten FR, Karin M. Immunity, inflammation, and cancer. Cell 2010;140:883-899.

9. Onodera T, Goseki N, Kosaki G. [Prognostic nutritional index in gastrointestinal surgery of malnourished cancer patients]. Nihon Geka Gakkai Zasshi 1984;85:1001-1005. Japanese.

10. Mohri Y, Inoue Y, Tanaka K, Hiro J, Uchida K, Kusunoki M. Prog- nostic nutritional index predicts postoperative outcome in colorectal cancer. World J Surg 2013;37:2688-2692.

11. Hong YM, Cho M, Yoon KT, Chu CW, Yang KH, Park YM, et al. Risk factors of early recurrence after curative hepatectomy in hepatocellular carcinoma. Tumour Biol 2017;39:1010428317720863.

12. Gabrielson A, Wu Y, Wang H, Jiang J, Kallakury B, Gatalica Z, et al. Intratumoral CD3 and CD8 T-cell densities associated with relapse-free survival in HCC. Cancer Immunol Res 2016;4:419-430.

13. Wu SJ, Lin YX, Ye H, Li FY, Xiong XZ, Cheng NS. Lymphocyte to monocyte ratio and prognostic nutritional index predict survival outcomes of hepatitis B virus-associated hepatocellular carcinoma patients after curative hepatectomy. J Surg Oncol 2016;114:202-210.

14. Liu Y, Wang ZX, Cao Y, Zhang G, Chen WB, Jiang CP. Preoperative inflammation-based markers predict early and late recurrence of hepatocellular carcinoma after curative hepatectomy. Hepatobiliary Pancreat Dis Int 2016;15:266-274.

15. Goh BK, Kam JH, Lee SY, Chan CY, Allen JC, Jeyaraj P, et al. Significance of neutrophil-to-lymphocyte ratio, platelet-to-lymphocyte ratio and prognostic nutrition index as preoperative predictors of early mortality after liver resection for huge $(\geq 10 \mathrm{~cm})$ hepatocellular carcinoma. J Surg Oncol 2016;113:621-627.

16. Ni XC, Yi Y, Fu YP, He HW, Cai XY, Wang JX, et al. Prognostic value of the modified Glasgow Prognostic Score in patients undergoing radical surgery for hepatocellular carcinoma. Medicine (Baltimore) 2015;94:e1486.

17. Schütte K, Tippelt B, Schulz C, Röhl FW, Feneberg A, Seidensticker R, et al. Malnutrition is a prognostic factor in patients with hepatocellular carcinoma (HCC). Clin Nutr 2015;34:1122-1127.

18. Huang TH, Hsieh CC, Kuo LM, Chang CC, Chen CH, Chi CC, et al. Malnutrition associated with an increased risk of postoperative complications following hepatectomy in patients with hepatocellular carcinoma. HPB (Oxford) 2019;21:1150-1155.

19. Man Z, Pang Q, Zhou L, Wang Y, Hu X, Yang S, et al. Prognostic significance of preoperative prognostic nutritional index in hepatocellular carcinoma: a meta-analysis. HPB (Oxford) 2018;20:888-895.

20. Wang Z, Wang J, Wang P. The prognostic value of prognostic nutritional index in hepatocellular carcinoma patients: a meta-analysis of observational studies. PLoS One 2018;13:e0202987.

21. Ji F, Liang Y, Fu S, Chen D, Cai X, Li S, et al. Prognostic value of combined preoperative prognostic nutritional index and body mass index in HCC after hepatectomy. HPB (Oxford) 2017;19:695-705.

22. Hatanaka T, Kakizaki S, Uehara D, Nagashima T, Ueno T, Namikawa $\mathrm{M}$, et al. Impact of the prognostic nutritional index on the survival of Japanese patients with hepatocellular carcinoma treated with sorafenib: a multicenter retrospective study. Intern Med 2019;58:18351844.

23. Zhang X, Li C, Wen T, Peng W, Yan L, Yang J. Postoperative prognostic nutritional index predicts survival of patients with hepatocellular carcinoma within milan criteria and hypersplenism. J Gastrointest Surg 2017;21:1626-1634.

24. Kim HJ, Kim CY, Park EK, Hur YH, Koh YS, Kim HJ, et al. Volumetric analysis and indocyanine green retention rate at $15 \mathrm{~min}$ as predictors of post-hepatectomy liver failure. HPB (Oxford) 2015;17:159-167. 